\title{
Temporal patterns of hospital readmissions according to disease category for patients after elective colorectal surgery
}

\author{
Fabian Grass MD ${ }^{1,2}$ (1) | Martin Hübner MD ${ }^{2}$ ｜ Jacopo Crippa MD ${ }^{1}$ | \\ Jenna K. Lovely PharmD, RPh, BCPS ${ }^{3}$ | Marianne Huebner PhD ${ }^{4}$ | \\ David W. Larson MD, MBA ${ }^{1}$
}

${ }^{1}$ Division of Colon and Rectal Surgery, Mayo Clinic, Rochester, Minnesota

${ }^{2}$ Department of Visceral Surgery, Lausanne University Hospital CHUV, University of Lausanne (UNIL), Lausanne, Switzerland

${ }^{3}$ Hospital Pharmacy Services, Mayo Clinic, Rochester, Minnesota

${ }^{4}$ Department of Health Sciences Research, Mayo Clinic, Rochester, Minnesota

\section{Correspondence}

David W. Larson, Division of Colon and Rectal Surgery, Mayo Clinic, 200 First Street SW, Rochester, MN 55905.

Email: larson.david2@mayo.edu

\begin{abstract}
Rationale: The aim of this study was to identify temporal readmission patterns according to baseline disease categories to provide opportunities for targeted interventions.

Methods: Retrospective analysis of consecutive adult ( $\geq 18$ years) patients who underwent elective colorectal resections (2011-2017) at Mayo Clinic Rochester, MN. A prospective administrative database including patient demographics, procedure characteristics, discharge information and specifics on 30-day readmissions (to index facility) including timing and reasons was utilized. The ICD-9 codes were regrouped into the main pathologies Cancer, Crohn's disease (CD)/chronic ulcerative colitis (CUC), and diverticular disease.

Results: In total, 521 (7.2\%) out of 7245 patients undergoing inpatient colorectal surgery were readmitted. In all increments of time from discharge (0-2 days: $31.3 \%$ of all readmissions, 3-7 days: $32.4 \%$ of all readmissions, $8-14$ days: $18 \%$ of all readmissions, and 15-30 days: $18.3 \%$ of all readmissions), reasons for readmission differed significantly (all $P<0.001$ ). Across all disease categories, early readmissions (within 2 days of discharge) were most likely due to ileus/obstruction (53.4\% of early readmissions), whereas with $42.5 \%$, infection was the most common cause for late readmissions ( $>7$ days). Patients with home discharge were more likely to be readmitted earlier within the 30-day observation period $(P=0.099)$, whereas patients with a longer length of index hospital stay ( $>7$ days) were readmitted later $(P=0.080)$.

Conclusions: Reasons for readmission appear to be universal across different disease categories. Targeted educational and collaborative measures may help to mitigate the burden of hospital readmissions to index facilities.
\end{abstract}

\section{KEYWORDS}

colorectal, disease category, readmission

\section{1 | INTRODUCTION}

In a value based healthcare market, institutions are focusing on reducing readmissions to improve quality and value of care while reducing financial penalties and societal scrutiny. ${ }^{1-3}$ Care standardization has been critical to lowering complication rates and shortening length of stay (LOS) in surgical patients. ${ }^{4}$ Several series have described patient characteristics, timing, or reasons for readmission within a standardized perioperative care practise. ${ }^{5-11}$ However, readmission patterns from the perspective of various disease categories have been less specifically 
studied. While several national registry analyses included disease categories as confounders to identify risk factors, specific assessment of patterns according to baseline indications was not performed ${ }^{3,12}$ Hechenbleikner et al used three different data collection methods in 735 institutional patients and revealed that both rates and reasons varied depending on the chosen methodologic approach of data collection rather than the disease category itself. ${ }^{13}$

The Nationwide Readmission Database arguably provides important insights from a national perspective. ${ }^{14,15}$ Nevertheless, large reports from individual centres are indispensable since deriving from more homogeneous patient cohorts with both standardized preoperative care and discharge practice. Furthermore, Mayo Clinic as a quaternary referral centre with nationwide patient accrual provides quite a unique setting. Collectively knowledge about disease-specific readmission patterns could provide further information to anticipate risk and optimize successful discharge preventing readmissions.

The aim of this study was to identify temporal readmission patterns broken down by baseline disease categories to provide opportunities for targeted interventions and to improve current standards of care.

\section{2 | METHODS}

This retrospective analysis was conducted for consecutive adult ( $\geq 18$ years) patients who underwent elective colorectal resections over a 7 year period (2011-2017) at Mayo Clinic, Rochester, MN. All patients were treated according to an enhanced recovery pathway (ERP), which became division standard of care as of 4 January $2011{ }^{16}$ The institutional ERP features standardized discharge criteria including pharmacist medication reconciliation with the surgical team. ${ }^{4,17}$ Discharge criteria included: tolerating oral diet, ambulating (at least 6 hours/day or return to baseline ambulation status), adequate pain control with oral medication, no evidence of complications by the time of discharge. A prospective administrative database including patient demographics, procedure characteristics, discharge information and specifics on readmissions (only to Mayo Clinic) including timing and reasons was utilized. Information on readmissions to other hospitals was not available. The protocol was approved by the local Institutional Review Board (\# 16-004192).

This study focused on patterns of readmission considering temporal aspects of different disease categories. International Classification of Disease, 9th Revision (ICD-9) procedure codes, along with patient information records were identified through both administrative and clinical data. The ICD-9 codes were regrouped into the following main pathologies: Cancer, Crohn's disease (CD)/chronic ulcerative colitis (CUC), diverticular disease, or other. Further details of the index hospital stay included LOS and discharge destination (home vs skilled nursing facility [SNF]). With each readmission, the diagnostic reason as retrieved through administrative data was regrouped into main categories infection (surgical site infection [SSI] or medical infectious complication), ileus/obstruction (paralytic or small bowel obstruction [SBO]), wound(wound dehiscence, wound management-related issues other than SSI) or stoma-related issues (including dehydration and skin irritation), medical reasons, bleeding complications (need for transfusion, including pelvic hematoma), and others.

\subsection{Statistical analysis}

Continuous variables were examined with histograms and summarized with mean and SD or median and interquartile range (IQR) for symmetric or skewed distributions, respectively. Categorical variables were described with frequencies and percentages. Comparisons were performed through nonparametric tests (Mann-Whitney) or two sample $t$ tests as appropriate.

LOS was defined as the number of calendar days from surgery to hospital discharge. Timing of readmission was calculated as days from discharge. For categorical variables Fisher's exact test was used. Surgical indication and reason for readmission was further subcategorized as groups of 0-2 (early readmission), 3-7 (first week), 8-14 (second week), and 15-30 (after 2 weeks) days. To illustrate the temporal aspects of readmission, cumulative incidence graphs were designed. A multigroup comparison across different disease categories was performed at 48 hours (early vs late readmissions).

Analyses were performed using the statistical software $\mathrm{R}$ version 3.3.1 and SAS 9.4. A two-sided $P$-value less than 0.05 was considered statistically significant.

\section{$3 \mid$ RESULTS}

In total, 521 (7.2\%) out of 7245 patients undergoing inpatient colorectal surgery were readmitted to the index facility within 30 days of discharge. While the median index LOS for patients without readmissions ( $n=6724)$ was 3 (IQR 2-6) days, it was 5 (IQR 3-7) days for readmitted patients $(P<0.001)$. Demographic details of readmitted patients are displayed in Table 1. Patients were readmitted at a median of 5 (IQR 2-11) days after discharge (Figure 1).

\section{1 $\mid$ Reasons for readmission}

In all increments of time from discharge (0-2 days: $31.3 \%$ of all readmissions, 3-7 days: $32.4 \%$, 8-14 days: $18.0 \%$, 15-30 days: $18.3 \%$ ), reasons for readmission differed significantly (all $P<0.001$, Figure 2). Early readmissions (within 2 days of discharge) were most likely due to ileus/obstruction, representing 87 (53.4\%) cases, whereas infection was the most common cause for late readmissions after 1 week (42.5\%). Patients with ileus/obstruction had a $47 \%$ probability of admission by day 2, a $72 \%$ probability of admission by day 7 and an $84 \%$ probability of admission by day 14 . Patients with infection had a $22 \%$ probability of admission by day 2 , a $56 \%$ probability of admission by day 7 and an $80 \%$ probability of admission by day 14 . Patients with wound/stoma complications had a $25 \%$ probability of admission by day 2 , a $66 \%$ probability of admission by day 7 and an $84 \%$ probability of admission by day 14. Figure S1 further details reasons for 
TABLE 1 Demographic details of readmitted patients

\begin{tabular}{|ll}
\hline Male (\%) & Total $(\mathbf{n}=\mathbf{5 2 1})$ \\
\hline Age (years) (median, IQR) & $286(54.9)$ \\
\hline Length of index hospital stay (median, IQR) & $55(38,67)$ \\
\hline $\begin{array}{l}\text { Days between discharge and } \\
\text { readmission (median, IQR) }\end{array}$ & $5(3,7)$ \\
\hline Initial diagnosis & $5(2,11)$ \\
\hline Crohn's/CUC & \\
Cancer/Neoplasia & $173(33.2)$ \\
Diverticular disease & $151(29.0)$ \\
Other & $148(28.4)$ \\
Surgical procedure & $49(9.4)$ \\
Segmental colectomy & \\
Ostomy creation & $168(32.2)$ \\
Rectal resection & $125(24)$ \\
Subtotal colectomy & $70(13.4)$ \\
Small bowel resection & $65(12.5)$ \\
IPAA & $46(8.8)$ \\
Other & $21(4)$ \\
Discharge to & $26(5.0)$ \\
Home & $136(26.1)$ \\
\hline SNF & $385(73.9)$ \\
\hline
\end{tabular}

Abbreviations: CUC, chronic ulcerative colitis; IPAA, ileal pouch anal anastomosis; IQR, interquartile range; SNF, skilled nursing facility.

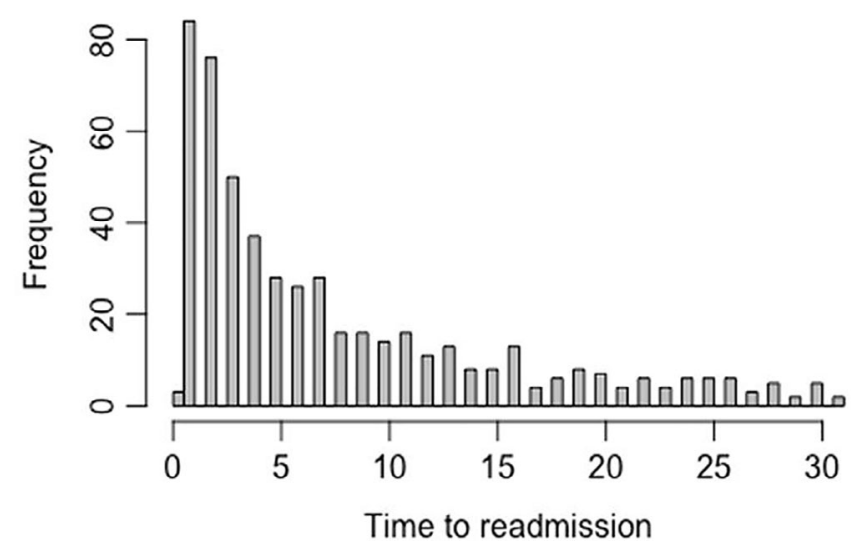

FIGURE 1 Time to readmission. Distribution of readmitted patients $(n=521)$ according to day after discharge from index hospital stay

readmission of readmitted patients according to different disease categories and increments of time.

\section{2 | Disease categories}

Figure 3 displays cumulative incidences of readmissions according to disease category. Readmitted cancer patients had a $25 \%$ probability

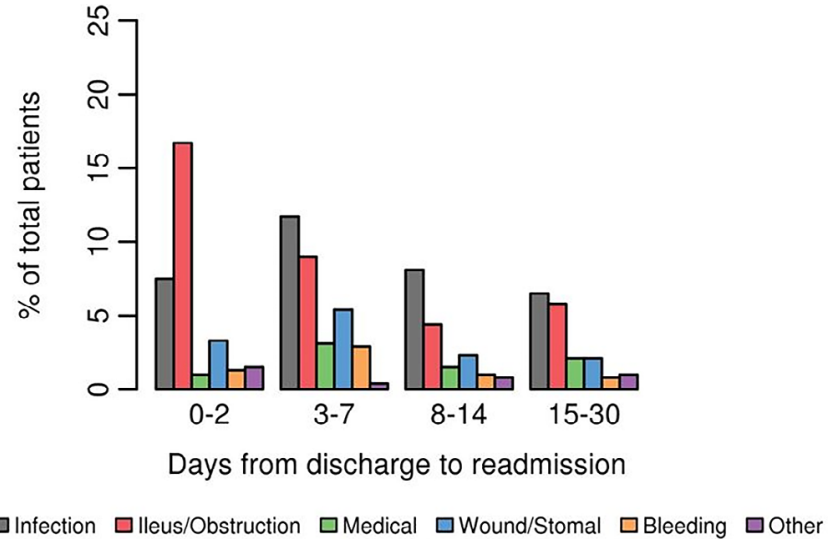

FIGURE 2 Reasons for readmission. Schematic representation of reasons for readmission according to timing of readmission (0-2 days, 3-7 days, second week, and after second week until 30 days)

of readmission within 2 days, with $50 \%$ of the readmissions by day 7 , and an $80 \%$ probability of readmission by day 14 . Patients with a diagnosis of diverticular disease had a $29 \%$ probability of readmission by day 2 , with $76 \%$ of the readmissions by day 7 , while $92 \%$ of patients were readmitted by day 14 . Crohn's disease/CUC patients had a $33 \%$ probability of readmission by day 2 , with $61 \%$ of the readmissions by day $7 ; 83 \%$ of readmissions occurred by day 14 . No statistical difference between early and late ( $>48$ hours) readmission was noted between patients with different disease category $(P=0.292)$. Furthermore, all disease categories followed a similar pattern with ileus being the most common reason for early readmission and infection the most common reason for late readmission.

Patients with home discharge were more likely to be readmitted earlier within the 30-day observation period ( $P=0.099$, Figure $4 A)$, whereas patients with a longer length of index hospital stay ( $>7$ days) were readmitted later $(P=0.080$, Figure 4B).

\section{4 | DISCUSSION}

This descriptive study revealed similar patterns and reasons for readmission independently of the underlying disease category. While ileus and obstruction were prevailing reasons for early readmission, late readmissions occurred mainly for SSIs. Stratification by disease category (cancer, inflammatory bowel disease [IBD] and diverticular disease) revealed further that patients undergoing surgery for diverticular disease were readmitted earlier ( $75 \%$ by day 7$)$ than patients with cancer or IBD ( $75 \%$ by day 11 , Figure 3$)$. Furthermore, a preponderance of early readmitted patients from home as opposed to skilled nursing facilities was observed. Another sensitive finding of this study is that most readmissions occur rather early (within 1 week of discharge) except for patients with long primary hospital stay (Figures 1 and $4 \mathrm{~B}$ ), with no difference between short and very short index stays.

This study focused on readmissions to the index facility solely to assure accuracy and consistency in assessment and categorization. 

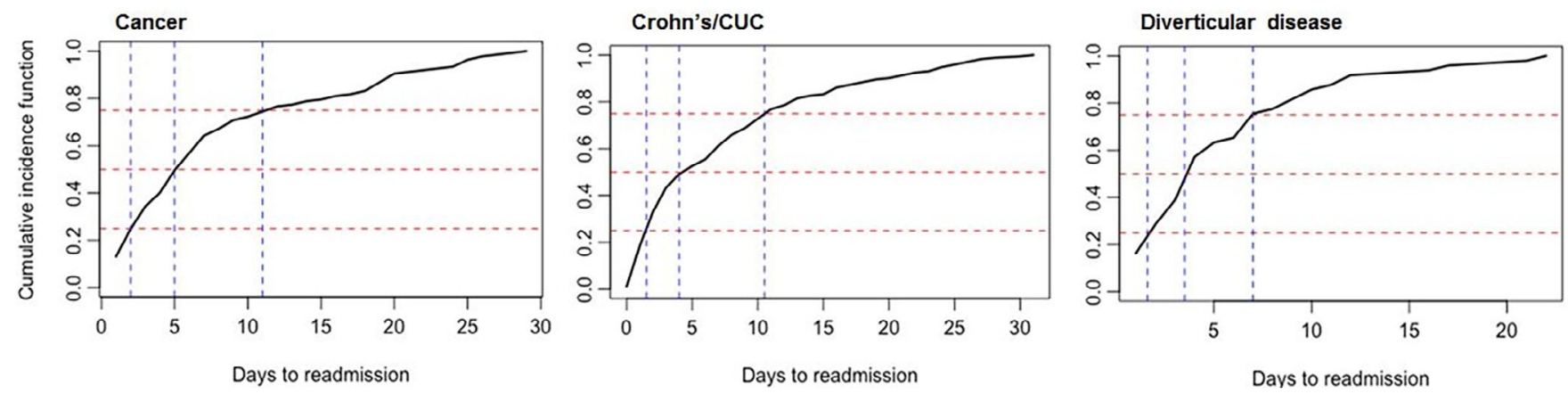

FIGURE 3 Cumulative incidence of readmission according to underlying disease. Cancer patients: $25 \%$ of the readmissions occur by day $2,50 \%$ of the readmissions by day $5,75 \%$ of the readmissions by day 11 , and $80 \%$ of the readmissions occur by day 15 . Crohn's/CUC patients: $25 \%$ of the readmissions occur by day $1.5,50 \%$ of the readmissions by day $4,75 \%$ of the readmissions by day 10.5 , and $80 \%$ of the readmissions occur by day 12 . Diverticular disease patients: $25 \%$ of the readmissions occur by day $2,50 \%$ of the readmissions by day $4,75 \%$ of the readmissions by day 7 , and $80 \%$ of the readmissions occur by day 9 . CUC, chronic ulcerative colitis

(A)

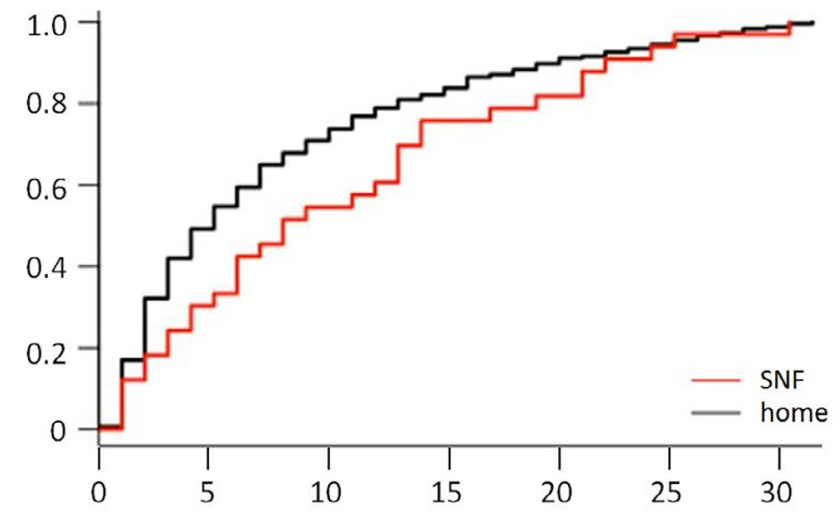

(B)

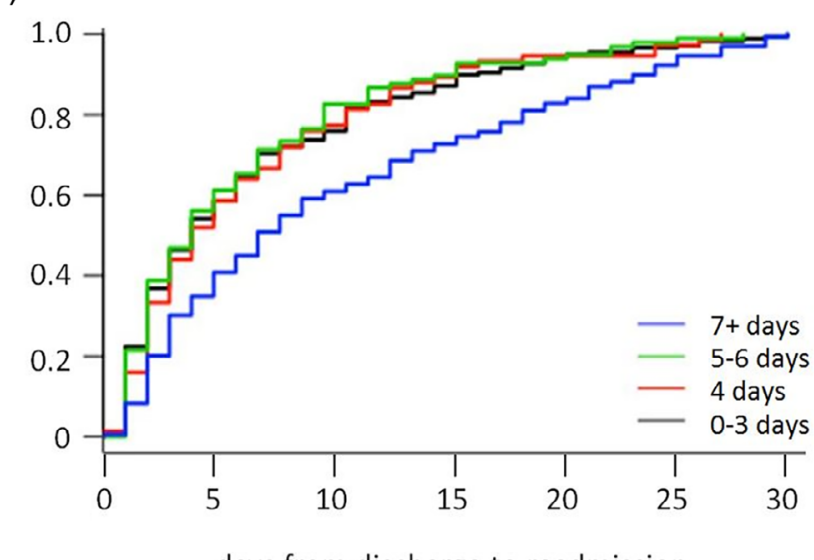

FIGURE 4 Cumulative incidence of readmitted patients $(n=521)$ according to, A, discharge location and B, length of primary hospital stay. SNF, skilled nursing facility

Overall, $7.91 \%$ of patients were readmitted within 30 days, which arguably may underestimate the actual readmission rate (including independent facilities) considering the quaternary referral setting of Mayo Clinic. The present study was not designed to predict hospital readmission or to identify risk factors for hospital readmission. However, it provides valuable data on timing and diagnostic reasons for readmission. Interestingly, reasons for readmission, broadly categorized into obstructive, infectious, stoma-related and medical, were consistent among disease categories. Thus, practise-relevant targeted strategies for all patients may help to decrease costly hospital readmissions.

Cancer and IBD patients were readmitted later in the postdischarge period, which may be related to a higher proportion of ostomates in these disease categories. Dehydration and electrolytic disturbances are common in patients with new ileostomies, and specific education including nutritional advice, thorough follow-up and a suspicious attitude towards dehydration-related symptoms are of utmost importance. Specialized outpatient follow-up in dedicated facilities (dehydration clinic) may thus need to be planned ahead in patients in whom ileostomy management is challenging during the index stay.

In line with previous evidence, shorter LOS in the setting of ERPs did not result in increased readmission rates. ${ }^{18,19}$ Across all surgical indications, ileus and obstructive symptoms remain the number one reason for early hospital readmission (Figure S1). The institutional pathway does not retain return of bowel function as mandatory discharge criteria. ${ }^{20}$ In the setting of shorter postoperative surveillance within the ERP, a suspicious attitude may help to recognize and manage ileus symptoms before discharge to avoid more costly readmissions to inpatient care. SSIs in a colorectal surgery practise are frequent and occur consistently any time over the 30-day observation period. While deep infections typically occur early and warrant invasive treatment and observation in an inpatient setting, superficial infections occurring later after discharge may be successfully treated by primary care physicians providing proper communication and coordination of care between clinicians of index and outpatient care 
teams. ${ }^{21}$ Coordination with primary care is of particular importance for a quaternary referral centre with nationwide accrual such as Mayo Clinic.

The retrospective study design and focus on readmissions to the index facility solely arguably represent limitations with potential underestimation of the overall readmission rate. This observational study did not intend to identify risk factors for hospital readmission but to study temporal aspects instead, aiming to provide new insights through longitudinal analysis of the 30-day post-discharge period. The focus on different indications and disease categories may thus provide new insights for potential improvements in care and patient expectations.

In conclusion, reasons for readmission appear to be universal across different disease categories. Targeted educational and collaborative measures may help to mitigate the burden of hospital readmissions to index facilities.

\section{CONFLICT OF INTEREST}

The authors declare no conflict of interest.

\section{ORCID}

Fabian Grass (ID) https://orcid.org/0000-0001-9884-2879

\section{REFERENCES}

1. Jha AK, Orav EJ, Epstein AM. Public reporting of discharge planning and rates of readmissions. N Engl J Med. 2009;361(27):2637-2645.

2. Zuckerman RB, Sheingold SH, Orav EJ, Ruhter J, Epstein AM. Readmissions, observation, and the hospital readmissions reduction program. N Engl J Med. 2016;374(16):1543-1551.

3. Damle RN, Cherng NB, Flahive JM, et al. Clinical and financial impact of hospital readmissions after colorectal resection: predictors, outcomes, and costs. Dis Colon Rectum. 2014;57(12):1421-1429.

4. Larson DW, Lovely JK, Cima RR, et al. Outcomes after implementation of a multimodal standard care pathway for laparoscopic colorectal surgery. Br J Surg. 2014;101(8):1023-1030.

5. Morris MS, Deierhoi RJ, Richman JS, Altom LK, Hawn MT. The relationship between timing of surgical complications and hospital readmission. JAMA Surg. 2014;149(4):348-354.

6. Al-Mazrou AM, Suradkar K, Mauro CM, Kiran RP. Characterization of readmission by day of rehospitalization after colorectal surgery. Dis Colon Rectum. 2017;60(2):202-212.

7. Francis NK, Mason J, Salib E, et al. Factors predicting 30-day readmission after laparoscopic colorectal cancer surgery within an enhanced recovery programme. Colorectal Dis. 2015;17(7):O148-0154.

8. Yuen A, Elnahas A, Azin A, Okrainec A, Jackson TD, Quereshy FA. Is expedited early discharge following elective surgery for colorectal cancer safe? An analysis of short-term outcomes. Surg Endosc. 2015.

9. Lawrence JK, Keller DS, Samia H, et al. Discharge within 24 to 72 hours of colorectal surgery is associated with low readmission rates when using enhanced recovery pathways. J Am Coll Surg. 2013; 216(3):390-394.

10. Shah PM, Johnston L, Sarosiek B, et al. Reducing readmissions while shortening length of stay: the positive impact of an enhanced recovery protocol in colorectal surgery. Dis Colon Rectum. 2017;60(2): 219-227.

11. Grass F, Ansell J, Petersen M, Mathis KL, Lightner AL. Risk factors for 90-day readmission and return to the operating room following abdominal operations for Crohn's disease. Surgery. 2019;166(6): 1068-1075.

12. Bartlett EK, Hoffman RL, Mahmoud NN, Karakousis GC, Kelz RR. Postdischarge occurrences after colorectal surgery happen early and are associated with dramatically increased rates of readmission. Dis Colon Rectum. 2014;57(11):1309-1316.

13. Hechenbleikner EM, Makary MA, Samarov DV, et al. Hospital readmission by method of data collection. J Am Coll Surg. 2013;216 (6):1150-1158.

14. Rattan R, Parreco J, Lindenmaier LB, et al. Underestimation of unplanned readmission after colorectal surgery: a national analysis. J Am Coll Surg. 2018;226(4):382-390.

15. Zafar SN, Shah AA, Nembhard C, et al. Readmissions after complex cancer surgery: analysis of the nationwide readmissions database. J Oncol Pract. 2018;14(6):e335-e345.

16. Lovely JK, Maxson PM, Jacob AK, et al. Case-matched series of enhanced versus standard recovery pathway in minimally invasive colorectal surgery. Br J Surg. 2012;99(1):120-126.

17. Lovely JK, Larson DW, Quast JM. A clinical practice agreement between pharmacists and surgeons streamlines medication management. Jt Comm J Qual Patient Saf. 2014;40(7):296-302.

18. Wood T, Aarts MA, Okrainec A, et al. Emergency room visits and readmissions following implementation of an enhanced recovery after surgery (iERAS) program. J Gastrointest Surg. 2018;22(2):259-266.

19. Liu VX, Rosas E, Hwang J, et al. Enhanced recovery after surgery program implementation in 2 surgical populations in an integrated health care delivery system. JAMA Surg. 2017;152(7):e171032.

20. Ellis CN. Discharge criteria after colon resection: is return of bowel function necessary? Am J Surg. 2017;213(3):544-547.

21. Martin D, Hubner M, Moulin E, et al. Timing, diagnosis, and treatment of surgical site infections after colonic surgery: prospective surveillance of 1263 patients. J Hosp Infect. 2018;100(4):393-399.

\section{SUPPORTING INFORMATION}

Additional supporting information may be found online in the Supporting Information section at the end of this article.

How to cite this article: Grass F, Hübner M, Crippa J, Lovely JK, Huebner M, Larson DW. Temporal patterns of hospital readmissions according to disease category for patients after elective colorectal surgery. J Eval Clin Pract. 2021;27:218-222. https://doi.org/10.1111/jep.13387 